\begin{tabular}{|c|c|}
\hline Title & Structural basis for pore forming mechanism of staphylococcal $\alpha$-hemolysin \\
\hline Author(s) & $\begin{array}{l}\text { Sugawara, T akaki; Y amashita, Daichi; Kato, Koji; Peng, Zhao; U eda, Junki; Kaneko, Jun; Kamio, Y oshiyuki; Tanaka, } \\
\text { Y oshikazu; Y ao, Min }\end{array}$ \\
\hline Citation & $\begin{array}{l}\text { Toxicon, 108, 226-231 } \\
\text { https://doi.org/10.1016/.toxicon.2015.09.033 }\end{array}$ \\
\hline Issue Date & 2015-12-15 \\
\hline Doc URL & http:/hdl.handle.net/2115/63926 \\
\hline Rights & $\begin{array}{l}\text { (c) 2015, Elsevier. Licensed under the Creative Commons A ttribution-NonCommercial-NoDerivatives 4.0 International } \\
\text { http://creativecommons.org/icenses/by-nc-nd/4.0/ }\end{array}$ \\
\hline Rights(URL) & http://creativecommons.org/icenses/by-nc-nd/4.0/ \\
\hline Tyре & article (author version) \\
\hline File Information & Final_RevisedManuscript.pdf \\
\hline
\end{tabular}

Instructions for use 


\section{Structural basis for pore-forming mechanism of staphylococcal $\alpha$-hemolysin}

Takaki Sugawara $^{1}$, Daichi Yamashita ${ }^{1}$, Koji Kato $^{1,2}$, Zhao Peng $^{3}$, Junki Ueda ${ }^{3}$, Jun Kaneko ${ }^{3}$, Yoshiyuki Kamio $^{3}$, Yoshikazu Tanaka ${ }^{1,2 *}$, and Min Yao ${ }^{1,2}$

1. Graduate School of Life Sciences, Hokkaido University, Sapporo, 060-0810, Japan.

2. Faculty of Advanced Life Sciences, Hokkaido University, Sapporo, 060-0810, Japan

3. Graduate School of Agricultural Science, Tohoku University, Sendai 981-8555, Japan

9

10 "To whom correspondence should be addressed.

11 Tel. \& Fax: +81-11-706-9017

12 E-mail: tanaka@sci.hokudai.ac.jp

13

14 Keywords: staphylococcal $\alpha$-hemolysin, pore-forming toxin, crystal structure 
Abstract

Staphylococcal $\alpha$-hemolysin $(\alpha-\mathrm{HL})$ is a $\beta$-barrel pore-forming toxin (PFT) expressed by

Staphylococcus aureus. $\alpha-\mathrm{HL}$ is secreted as a water-soluble monomeric protein, which binds to target

membranes and forms membrane-inserted heptameric pores. To explore the pore-forming

mechanism of $\alpha$-HL in detail, we determined the crystal structure of the $\alpha$-HL monomer and prepore

using H35A mutant and W179A/R200A mutant, respectively. Although the overall structure of the monomer was similar to that of other staphylococcal PFTs, a marked difference was observed in the

23 N-terminal amino latch, which bent toward the prestem. Moreover, the prestem was fastened by the cap domain with a key hydrogen bond between Asp45 and Tyr118. Prepore structure showed that the transmembrane region is roughly formed with flexibility, although the upper half of the $\beta$-barrel is formed appropriately. Structure comparison among monomer, prepore and pore revealed a series of motions, in which the N-terminal amino latch released upon oligomerization destroys its own key hydrogen bond betweem Asp45-Try118. This action initiated the protrusion of the prestem. Y118F mutant and the N-terminal truncated mutant markedly decreased in the hemolytic activity, indicating the importance of the key hydrogen bond and the N-terminal amino latch on the pore formation. Based on these observations, we proposed a dynamic molecular mechanism of pore formation for $\alpha$-HL. 


\section{$35 \quad$ Keywords}

36 staphylococcal $\alpha$-hemolysin, pore-forming toxin, crystal structure

\section{$37 \quad$ Abbreviations}

$38 \quad \alpha$-HL: $\alpha$-hemolysin, PFT: pore-forming toxin 


\section{$40 \quad$ Highlights}

- Crystal structures of the $\alpha$-HL monomer and prepore were derermined.

- The prestem is fastened by a key hydrogen bond between Asp45 and Tyr118 in monomer.

- In prepore, the transmembrane region is roughly formed with flexibility.

- Upon oligomerization, the released amino latch destroys the key interaction to release prestem.

- A dynamic pore-forming mechanism, where the amino latch plays a key role, is proposed. 


\section{Introduction}

Pathogenic bacteria secrete pore-forming toxins (PFTs) for attacking target cells. PFTs are

secreted as water-soluble monomeric proteins, which assemble on the target cells for forming

membrane-inserted pores. These pores then lead to cell death. PFTs are classified into two families

according to the secondary structure of the transmembrane region, i.e., $\alpha$-PFTs and $\beta$-PFTs.

Staphylococcus aureus, a major cause of hospital- and community-acquired infections, expresses

several $\beta$-PFTs, including $\alpha$-hemolysin $(\alpha$-HL), $\gamma$-hemolysin $(\gamma$-HL), and leukocidin (LUK), for

killing blood cells (Kaneko and Kamio, 2004). $\alpha$-HL is a mono-component heptameric $\beta$-PFT, while

the other two PFTs are bi-component octameric $\beta$-PFTs composed of two homologous polypeptides

( $\sim 30 \%$ of amino acid sequence identity), designated as $\mathrm{F}$ and $\mathrm{S}$ components, respectively. $\alpha-\mathrm{HL}$ reveals $\sim 30 \%$ and $\sim 20 \%$ sequence identity with $\mathrm{F}$ and $\mathrm{S}$ components, respectively.

Previous biochemical experiments have suggested a general pore-forming mechanism, in

which the soluble monomeric components assemble into a ring-shaped pore via a nonlytic oligomeric

61 intermediate known as a prepore (Kawate and Gouaux, 2003; Nguyen et al., 2002; Walker et al.,

1995). However, the underlying mechanisms remain unelucidated because of the unavailability of

63 the crystal structure of the $\alpha$-HL monomer without an artificial binder and prepore. To explore this and W179A/R200A mutant ( $\alpha$-HL-WR), which revealed monomeric and prepore form, respectively. 
$67 \alpha$-HL assembly using hydrophobic interactions, in which the amino latch plays a key role, was

68 proposed.

69 


\section{Materials and methods}

\subsection{Cloning, expression, and purification}

The expression vector for mutants of $\alpha$-HL was prepared by site-directed mutagenesis using

the expression plasmid of wild-type $\alpha-\mathrm{HL}$ as the template. In the resultant expression vector, a

His $_{6}$-tag was fused at the $\mathrm{C}$ terminus of the toxin. Expression and purification of these mutants were performed as previously described (Sugawara et al., 2013; Tanaka et al., 2011). In brief, the

Escherichia coli strain B834 (DE3) harboring the desired plasmid was grown at $37^{\circ} \mathrm{C}$ in $\mathrm{LB}$ medium containing $25 \mu \mathrm{g} / \mathrm{mL}$ of kanamycin. Isopropyl- $\beta$-D-thiogalactoside was added to obtain a final concentration of $0.5 \mathrm{mM}$ when the optical density at $600 \mathrm{~nm}$ reached $0.6-0.8$, followed by continuous culture for $24 \mathrm{~h}$. The cells were collected and then disrupted by sonication in the sonication buffer (20 mM Tris- $\mathrm{HCl}, \mathrm{pH} 8.0$, and $200 \mathrm{mM} \mathrm{NaCl}$ ). The $\alpha$-HL mutants present in the supernatant were purified on a His-trap affinity column and a HiLoad 26/60 Superdex 200 column (GE Healthcare, Buckinghamshire, UK).

\subsection{Crystallization, X-ray diffraction data collection, and structure determination} Crystals of $\alpha$-HL-H35A suitable for further experiments were grown from a buffer

containing $0.05 \mathrm{M}$ sodium cacodylate ( $\mathrm{pH}$ 6.5), $18 \mathrm{mM}$ calcium chloride, $2.5 \mathrm{mM}$ spermine, $9 \%$

(v/v) 2-propanol, and 5\% (w/v) ethylene glycol. Crystals of $\alpha$-HL-WR were grown from a buffer containing 40\% MPD and $0.1 \mathrm{M}$ citric acid ( $\mathrm{pH}$ 4.0). X-ray diffraction experiments were performed at Photon Factory (Tsukuba, Japan) and SPring-8 (Harima, Japan) under proposals 
2012G515/2014G022 and 2012A1179/2012B1215/2013A1115/2015A1117, respectively. Moreover,

X-ray diffraction dataset of $\alpha-\mathrm{HL}-\mathrm{H} 35 \mathrm{~A}$ and $\alpha$-HL-WR was collected on the beamline BL5A and

BL17A at Photon Factory, respectively. The diffraction data were indexed, integrated, scaled, and merged using the XDS program (Kabsch, 2010). The data statistics are shown in Table 1. Crystal structures were determined by the molecular replacement method using the PHASER software (McCoy et al., 2007). The structure of $\alpha$-HL pore (PDB ID 3ANZ) was used as a search probe. To monitor the refinement, a random 5\% subset was set aside for the calculation of the $\mathrm{R}_{\text {free }}$ factor. After rigid body refinement and manual model building with COOT (Emsley et al., 2010), individual atomic coordinate refinement and individual ADP refinement were performed with phenix.refine (Adams et al., 2010). The refinement statistics are summarized in Table 1. The atomic coordinates of $\alpha-H L-H 35 A$ and $\alpha-H L-W R$ have been deposited in the Protein Data Bank, www.pdb.org (PDB ID code 4YHD and 4P24, respectively).

2.3 Assays of hemolytic activity and toxin binding to the rabbit erythrocytes.

Aliquots of washed $1 \mathrm{ml}$ of $1 \%$ rabbit erythrocytes were incubated with $8 \mu \mathrm{g}$ of $\alpha$-HL or its mutants at $37^{\circ} \mathrm{C}$ for $30 \mathrm{~min}$. After centrifugation, the supernatant was assayed for hemoglobin at 541 $\mathrm{nm}$. Toxin binding to the rabbit erythrocyte membrane was analyzed by Western blotting with anti- $\alpha-H L$ antiserum as described previously (Kaneko et al., 1997). For the detection of heptermar complex, toxin treated rabbit erythrocyte membranes were solubilized with $1 \%$ SDS at 20 or $95^{\circ} \mathrm{C}$, 
109 and then analyzed by Western blotting (Kaneko et al., 1997).

110

111 


\section{Results and discussion}

\subsection{Crystal structure of $\alpha-H L-H 35 A$ reveals key hydrogen bond between Asp 45 and Try 118}

The substitution of His35 causes marked decreases in oligomerization and hemolysis activities and leads to an insufficient cell-binding activity (Walker and Bayley, 1995). As His35 is located at the interface between the protomers, this mutant is likely to retain the monomeric structure; however, in the absence of appropriate interprotomer interactions, it may not form heptamers. In the present study, we determined the crystal structure of $\alpha-\mathrm{HL}-\mathrm{H} 35 \mathrm{~A}$ mutants at a resolution of $2.80 \AA$.

As expected, the revealed structure was an $\alpha$-HL monomer, in which the prestem was folded beside the cap domain (Fig. 1A). The prestem region, which is extended in the pore to form a $\beta$-barrel, was folded into a three-stranded antiparallel $\beta$-sheet with a long connecting loop in the monomer. Furthermore, a large conformational change was observed in the amino latch. Although the amino latch protrudes and interacts with the adjacent protomer in the pore, it is located at the edge of the $\beta$-sheet of the stem region. The tip of the amino latch forms a short helix in all known pore structures. However, this region had an extended conformation in the monomer.

The overall structure of the $\alpha$-HL monomer was similar to that of other staphylococcal PFT monomers; RMSD was $1.30 \AA$ for LukF, $1.14 \AA$ for LukF-PV, $1.10 \AA$ for LukD, $1.06 \AA$ for LukS-PV, and $0.91 \AA$ for Hlg2. The folded prestem was fastened by a loop located at the top of the cap domain (hereafter loop-A (Yamashita et al., 2011), Yamashita et al. 2014). Asp45 of the loop-A formed a hydrogen bond with Tyr118 of prestem (Fig. 1B). A part of the long loop of the prestem (Thr129- 
132 Gly134), involved in the formation of the transmembrane region of the pore, was disordered. These

133 structural characteristics are commonly observed in other staphylococcal PFT monomers. In contrast to these common structural features, marked differences were observed in the orientation of N-terminal amino latch and long connecting loop in the prestem (Fig. 1C). The amino latch of the $\alpha$-HL monomer bends toward the prestem, whereas it is aligned adjacent to the $\beta$-sheet in other PFTs (Fig. 1C). Consequently, only a short $\beta$-strand was formed in this region of the $\alpha$-HL monomer. Two hydrophobic residues (Ile5 and Ile7) of the bent N-terminus amino latch form a hydrophobic core with Phe39 located at the surface of the cap domain. This formation may supply the driving force for the bent conformation of the amino latch. These residues are not conserved in any other staphylococcal PFTs (Supplementary Fig. S1). Therefore, the hydrophobic core formation characteristics were common for all six $\alpha$-HL monomers in an asymmetric unit, they are possibly the

144 intrinsic conformations of $\alpha-\mathrm{HL}$ and not casual conformations because of flexibility. 
151 forms stable prepore state oligomer. To acquire knowledge about prepore of $\alpha$-HL, we determined 152 the crystal structure of this mutant in the presence of high concentration of MPD which induces 153 spontaneous heptameric assembly of $\alpha-\mathrm{HL}$ (Tanaka et al., 2011). The revealed structure was quite 154 similar to that of $\alpha$-HL pore (Fig. 3A). However, the electron density of the transmembrane region 155 was markedly ambiguous despite the clear density of the extramembrane half (Fig. 3B). The B-factor 156 of the transmembrane $\beta$-barrel is extraordinarily high $\left(>100 \AA^{2}\right)$ for this structure (Fig. 3A). These 157 observations suggest that the transmembrane region of $\alpha$-HL-WR is roughly formed with flexibility, 158 although the upper half of the $\beta$-barrel is formed appropriately. This result is consistent with the 159 previously determined prepore structure of staphylococcal two-component PFTs, i.e. prepore and 160 pore is different in the flexibility of the transmembrane region, although other regions are quite similar (Yamashita et al., 2014). Based on these observations, we consider the heptameric structure of $\alpha$-HL-WR as prepore of $\alpha$-HL, hereafter. Due to the absence of Trp179 and Arg200 responsible for the binding with the head group of phospholipid, the spontaneous pore formation of $\alpha-\mathrm{HL}-\mathrm{WR}$ 164 induced by MPD was likely to be incomplete, although their precise role on the $\beta$-barrel formation was not clarified from crystal structure. For staphylococcal two-component PFTs, the two-step pore-forming mechanism has been proposed, in which the upper part of the extramembrane domain of the $\beta$-barrel is formed first, followed by the formation of the bottom transmembrane part of this structure (Yamashita et al., 2014). The common propensity between the $\alpha$-HL prepore and that of 
170 pore-forming mechanism is also applicable to $\alpha$-HL.

\subsection{Mechanism to release the prestem using the $N$-terminal amino latch}

Our study presents the crystal structure of $\alpha-\mathrm{HL}$ in the monomeric and oligomeric prepore states, enabling the description of a dynamic mechanism of $\alpha-\mathrm{HL}$ assembly. Fig. 4A reveals the superposition of a monomer onto a protomer of the pore, which illustrates the motion of the released amino latch. The amino latch moves to the region previously occupied by the prestem. It is noteworthy that Asp13-Gly15 of amino latch in the pore occupies the position of Tyr118 in the monomer. Tyr118 is a key residue for fastening the prestem to the cap domain (Fig. 1B). These observations indicate that the released amino latch forces off the prestem by destroying the key latch, two mutants, i.e. Y118F mutant and truncate mutant of the N-terminal 14 residues activity comparable with the wild type (Fig. 2B). The Y118F mutant is plausibly unable to hold the prestem stably in the monomeric state due to the disappearance of the key hydrogen bond, and the 
189 the $\alpha-H L-\Delta$ N14 could bind to erythrocytes but diminished its pore formation activity. $\alpha-H L-\Delta$ N14 is 190 plausibly incapable to cleave the key hydrogen bond effectively because of the disappearance of the 191 N-terminal 14 residues. These result strongly supports the importance of physical contact in this 192 region.

Fig. 4B shows the superposition of two monomers on the two neighboring protomers of the clash was observed between the amino latch of one protomer (shown in green in Fig. 4B) and the prestem of the other (shown in red). The bent conformation of the amino latch, which is a structural characteristic peculiar to $\alpha-\mathrm{HL}$, substantiates the degree of the steric clash. The bent amino latch would be released by the steric repulsion when a monomer assembles with its adjacent protomer, which would subsequently release the prestem itself because of the abovementioned physical contact.

200 Furthermore, the substitution of the $\alpha$-HL amino latch with the amino latch of LukF, which had an extended conformation, caused a drastic loss of hemolytic activity (Jayasinghe et al., 2006). This report also indicates the importance of the steric repulsion of the amino latch owing to the bent conformation. In addition to the steric hindrance between the amino latch and prestem, hindrances 204 between the prestems of the two protomers were also observed (red and yellow in Fig. 4B). This 205 phenomenon would also contribute to the release of the prestem. 
209 help of hydrophobic core formation using Ile5 and Ile7 of the amino latch and Phe39 located at the 210 surface of the cap domain (Fig. 5A). In pore assembly, the amino latch protrudes and interacts with 211 the adjacent protomer (Fig. 5B). During this conformational change, the N-terminus region of the 212 amino latch folds into a short helix. This helix then forms a hydrophobic core with Ile14, Ile43, 213 Leu52, Val54, and Val231 of the adjacent protomer, which stabilizes the conformation of the long 214 protruded amino latch in the pore state (Fig. 5B). It is noteworthy that Ile5 and Ile7, important for the 215 bent conformation of the amino latch in the monomer, re-participate in hydrophobic interactions. 216 These two hydrophobic residues are important for the conformational conversion of the amino latch 217 from monomer to oligomer. In the monomer, the prestem covers the hydrophobic surface of the cap 218 domain, which binds to the N-terminal short helix. Further, the hydrophobic surface exposed by the 219 release of prestem binds to the hydrophobic amino latch of the adjacent protomer in the pore. 220 Altogether, $\alpha$-HL efficiently uses the hydrophobicity of the amino latch for its structural conversion. 221 Because of the stabilization of the protruded conformation in the pore, the amino latch may promote 222 the cleavage of the hydrogen bond fastening the prestem (Asp45-Try118), which would ensure the 223 release of the prestem. At the same time, this conformation would prevent the refolding of the stem 224 following its release. The decrease in the hemolytic activity by the truncation of the amino latch may 225 be partly caused by the inability to stably assemble into this conformation. 

mechanism of $\alpha$-HL was revealed. The prestem is fastened by a key hydrogen bond between Asp45 230 and Try118 in monomer, and the N-terminal amino latch released upon assembly destroys the key 231 hydrogen bond to release the prestem. During these processes, the hydrophobic interaction by the 232 N-terminal amino latch acts as key role. The $\beta$-barrel is formed by two-step manner as observed for 233 the previously reported staphylococcal two-component PFTs. The upper extaramembrane half is 234 formed in the prepore state, and the transmembrane region is inserted into the membrane to form a 235 pore, which completes the pore formation.

238 Acknowledgment X-ray diffraction experiments were performed at Photon Factory and SPring- 8 under

proposals 2012G515/2014G022 and 2012A1179/2012B1215/2013A1115/2015A1117, respectively.

241 This work was supported by Grants-in-Aid from the Ministry of Education, Science, Sports and 242 Culture of Japan (Y. T.).

245 Fig. 1 Crystals of $\boldsymbol{\alpha}$-hemolysin H35A monomer (A) The overall structure of the a-HL monomer 
246 (left) and protomer of the pore (right). The amino latch, prestem, cap, and rim are colored blue, 247 orange, green, and magenta, respectively. Loop-A in the monomer is shown in red. The disordered 248 region of the prestem is shown as an orange dotted line. (B) Interaction between the Loop-A and 249 prestem through a hydrogen bond between Asp45 and Tyr118. Asp45 and Tyr118 are shown as 250 sticks. The hydrogen bond is shown as a red dotted line. Colors of the cartoon correspond to Fig. 1A. 251 (C) Comparison of the monomeric structures of LukF and LukD (stereo view). For clarity, the amino 252 latch and prestem, which show marked structural differences between the three proteins, are 253 highlighted, and the cap and rim are all colored white.

Fig. 2 Hemolytic activity and erythrocyte binding activity of mutants. (A) Relative hemolytic

activity of wild-type $\alpha$-HL (WT), Y118F, $\Delta$ N14, and WR for rabbit erythrocytes. (B) Relative

binding amount of mutant proteins for the rabbit erythrocytes membrane. The graphs show average

values from three individual experiments and the corresponding SD. (C) Heptamer complex

259 formation of wild-type $\alpha-H L$ and WR mutant on rabbit erythrocyte membrane. Samples treated with $2601 \% \mathrm{SDS}$ at $100^{\circ} \mathrm{C}$ or $20^{\circ} \mathrm{C}$ were analyzed by Western blotting (shown as 100 and 20 , respectively). 261 Bands for monomer and complex form were indicated by white and black triangle, respectively.

Fig. 3 Structure of $\boldsymbol{\alpha}$-HL-WR mutant. (A) Tube model of the overall structure of heptameric 
width of the tube also corresponds to the B-factor. (B) Stereo representation of $2 \mathrm{Fo}-\mathrm{Fc}$ electron

268 Fig. 4 Steric hindrance of the amino latch (A) Stereo representation of the monomer superposed 269 on a protomer of the pore. Amino latch of the monomer and pore are shown as blue and green, 270 respectively. Prestem and stem are shown as yellow and red, respectively. The cap domain of the 271 monomer and pore are shown as white and pale blue, respectively. For clarity, the top of the protruding stem is truncated. (B) Stereo representation of monomers aligned as two adjacent protomers in the pore. The amino latch of each monomer is colored blue and green. The prestems are colored red and yellow. The cap and rim of each protomer are shown in white and pale blue.

Fig. 5 Hydrophobic interaction of the amino latch in the monomer (A) and in the pore (B)

Residues participating in the interaction are shown as yellow sticks. (A) Domain colors correspond to to the protomer; the yellow and red residues are derived from blue and green protomers, respectively. 


\begin{tabular}{|c|c|c|}
\hline & $\alpha-H L-H 35 A$ (4YHD) & $\alpha-H L-W R(4 P 24)$ \\
\hline \multicolumn{3}{|l|}{ Data collection } \\
\hline Beamline & Photon Factory BL5A & Photon Factory BL17A \\
\hline Space group & $P 2_{1}$ & $P 4{ }_{1} 2{ }_{1}$ \\
\hline \multicolumn{3}{|l|}{ Cell dimensions } \\
\hline $\mathrm{a}, \mathrm{b}, \mathrm{c}(\AA)$ & $75.9,128.9,135.3$ & $170.1,170.1,202.9$ \\
\hline$\alpha, \beta, \gamma\left(^{\circ}\right)$ & $90.0,91.6,90.0$ & $90,90,90$ \\
\hline Wavelength $(\AA)$ & 1.0 & 0.98 \\
\hline Resolution $(\AA)^{a}$ & $37.4-2.80(2.90-2.80)$ & $48.6-3.10(3.21-3.10)$ \\
\hline No of toto1/uniaun rofloctions & $222,318 / 61,440$ & $446,837 / 54,499$ \\
\hline No. or lotal/ untque ienections & $(25,460 / 7,895)$ & $(44,367 / 5,383)$ \\
\hline$R_{\mathrm{sym}}(\%)^{a, b}$ & $12.1(52.7)$ & $20.54(99.13)$ \\
\hline Completeness $(\%)^{a}$ & $95.8(76.7)$ & $99.99(99.98)$ \\
\hline Multiplicity $^{a}$ & $3.6(3.2)$ & $8.2(8.2)$ \\
\hline Average $I / \sigma(I)^{a}$ & $10.00(2.13)$ & $10.8(2.13)$ \\
\hline \multicolumn{3}{|l|}{ Refinement } \\
\hline Resolution $(\AA)$ & $37.4-2.80$ & $48.6-3.10$ \\
\hline $\mathrm{R}_{\text {work }} / \mathrm{R}_{\text {free }}$ & $0.215 / 0.263$ & $0.219 / 0.245$ \\
\hline \multicolumn{3}{|l|}{ No. of atoms } \\
\hline Protein & 13499 & 16232 \\
\hline Ligand/ion & 5 & 104 \\
\hline \multicolumn{3}{|l|}{ B-factors $\left(\AA^{2}\right)$} \\
\hline Protein & 40.1 & 60.7 \\
\hline Ligand/ion & 34.3 & 101.4 \\
\hline \multicolumn{3}{|l|}{ r.m.s.d. } \\
\hline Bond lengths $(\AA)$ & 0.004 & 0.005 \\
\hline Bond angles $\left({ }^{\circ}\right)$ & 0.78 & 1.04 \\
\hline
\end{tabular}

$283 a$ Values in parentheses correspond to the highest resolution shell.

$284 \quad b \quad R_{\text {merge }}=\Sigma_{\mathrm{h}} \Sigma_{\mathrm{i}}\left|I_{\mathrm{h}, \mathrm{i}}-<I_{\mathrm{h}}>\right| \Sigma_{\mathrm{h}} \Sigma_{\mathrm{i}}\left|I_{\mathrm{h}, \mathrm{i}}\right|$, where $<I_{\mathrm{h}}>$ is the mean intensity of a set of equivalent 285 reflections. 


\section{References}

288 Adams, P.D., Afonine, P.V., Bunkoczi, G., Chen, V.B., Davis, I.W., Echols, N., Headd, J.J., Hung, 289 L.W., Kapral, G.J., Grosse-Kunstleve, R.W., McCoy, A.J., Moriarty, N.W., Oeffner, R., Read, R.J., 290 Richardson, D.C., Richardson, J.S., Terwilliger, T.C., Zwart, P.H., 2010. PHENIX: a comprehensive 291 Python-based system for macromolecular structure solution. Acta crystallographica. Section D, 292 Biological crystallography 66, 213-221.

293 Emsley, P., Lohkamp, B., Scott, W.G., Cowtan, K., 2010. Features and development of Coot. Acta 294 crystallographica. Section D, Biological crystallography 66, 486-501.

295 Jayasinghe, L., Miles, G., Bayley, H., 2006. Role of the amino latch of staphylococcal 296 alpha-hemolysin in pore formation: a co-operative interaction between the $\mathrm{N}$ terminus and position 297 217. J Biol Chem 281, 2195-2204.

298 Kabsch, W., 2010. Xds. Acta Crystallogr D Biol Crystallogr 66, 125-132.

299 Kaneko, J., Kamio, Y., 2004. Bacterial two-component and hetero-heptameric pore-forming cytolytic 300 toxins: structures, pore-forming mechanism, and organization of the genes. Biosci Biotechnol 301 Biochem 68, 981-1003.

302 Kaneko, J., Ozawa, T., Tomita, T., Kamio, Y., 1997. Sequential binding of Staphylococcal 303 gamma-hemolysin to human erythrocytes and complex formation of the hemolysin on the cell 304 surface. Biosci Biotechnol Biochem 61, 846-851.

305 Kawate, T., Gouaux, E., 2003. Arresting and releasing Staphylococcal alpha-hemolysin at 
intermediate stages of pore formation by engineered disulfide bonds. Protein Sci 12, 997-1006.

Nguyen, V.T., Higuchi, H., Kamio, Y., 2002. Controlling pore assembly of staphylococcal gamma-haemolysin by low temperature and by disulphide bond formation in double-cysteine LukF mutants. Mol Microbiol 45, 1485-1498.

Sugawara, T., Yamashita, D., Tanaka, Y., Kaneko, J., Kamio, Y., Tanaka, I., Yao, M., 2013. Preliminary X-ray crystallographic study of staphylococcal alpha-haemolysin monomer. Acta crystallographica. Section F, Structural biology and crystallization communications 69 , 868-870. induces spontaneous assembly of staphylococcal $\alpha$-hemolysin into heptameric pore structure. Protein Science 20, 448-456.

Walker, B., Bayley, H., 1995. Key residues for membrane binding, oligomerization, and pore forming activity of staphylococcal alpha-hemolysin identified by cysteine scanning mutagenesis and targeted chemical modification. J Biol Chem 270, 23065-23071.

Walker, B., Braha, O., Cheley, S., Bayley, H., 1995. An intermediate in the assembly of a 323 Yamashita, D., Sugawara, T., Takeshita, M., Kaneko, J., Kamio, Y., Tanaka, I., Tanaka, Y., Yao, M., 
325 toxins. Nat Commun 5, 4897.

326 Yamashita, K., Kawai, Y., Tanaka, Y., Hirano, N., Kaneko, J., Tomita, N., Ohta, M., Kamio, Y., Yao, 327 M., Tanaka, I., 2011. Crystal structure of the octameric pore of staphylococcal gamma-hemolysin 328 reveals the beta-barrel pore formation mechanism by two components. Proceedings of the National 329 Academy of Sciences of the United States of America 108, 17314-17319.

330 331 


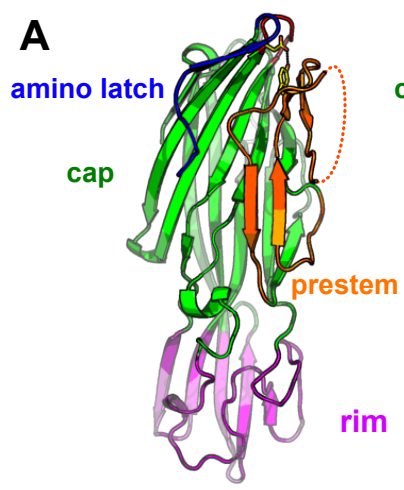

monomer
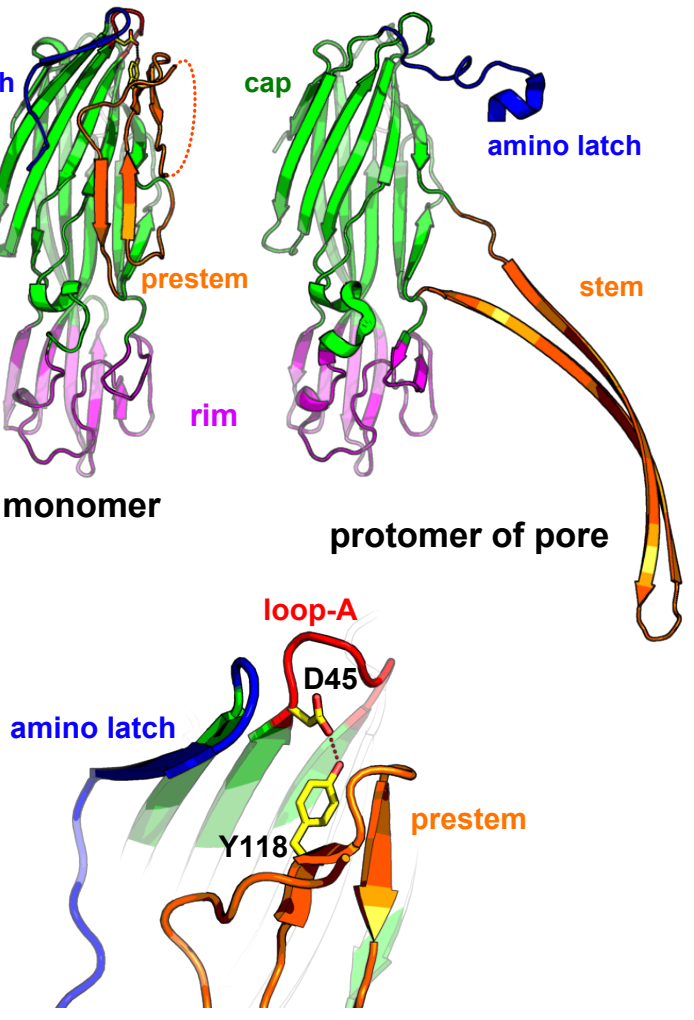

C

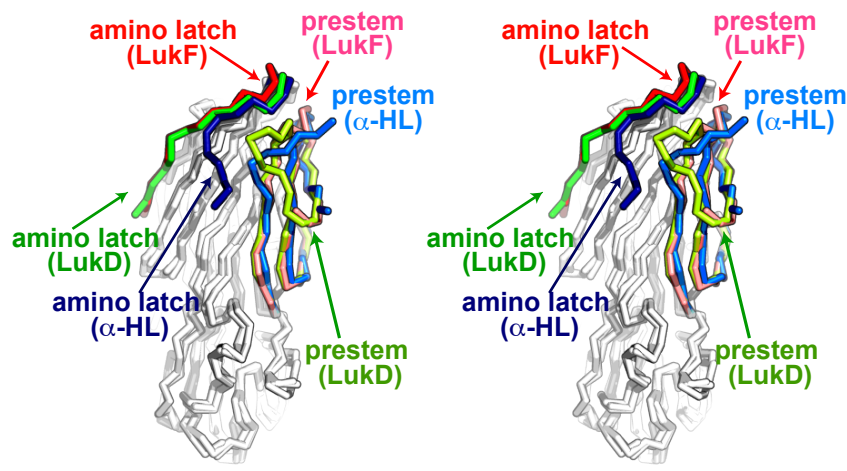

Fig. 1 (Sugawara et al.) 
A

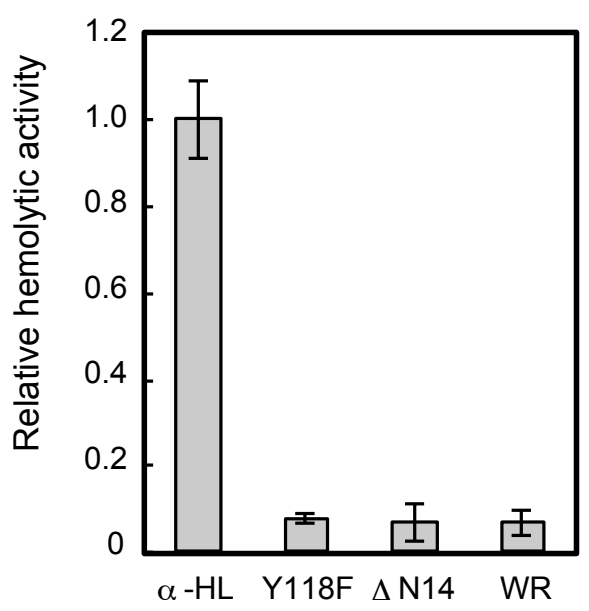

B

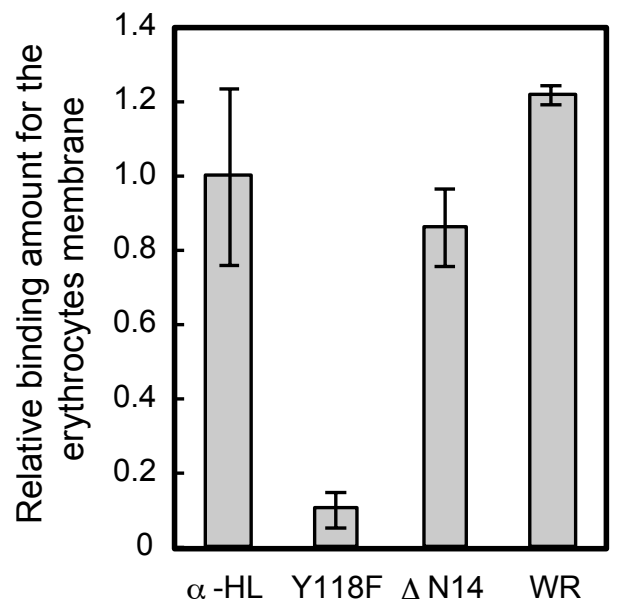

C

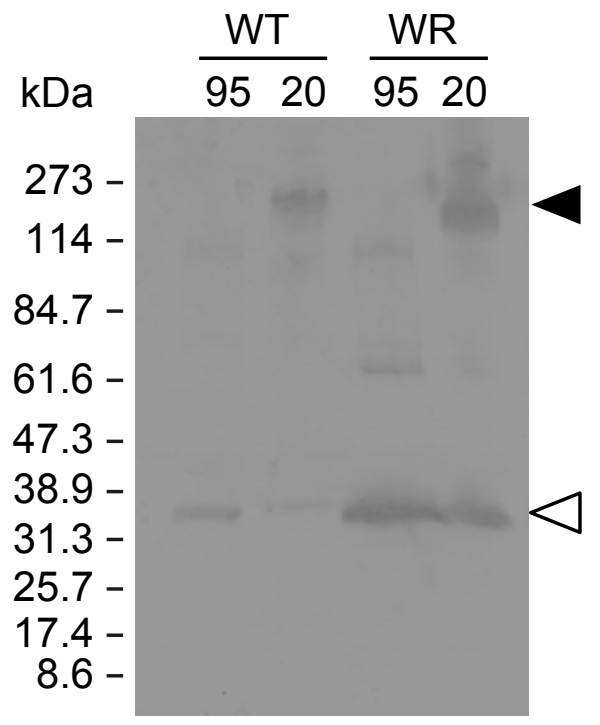

Fig. 2 (Sugawara et al.) 
A

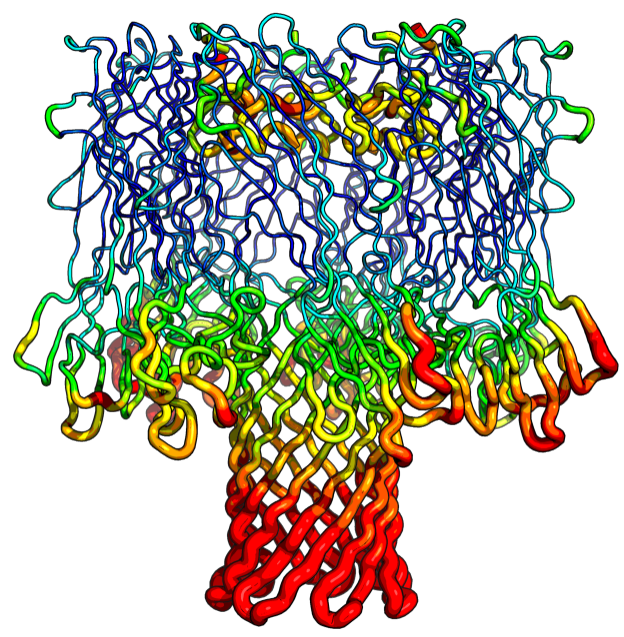

B
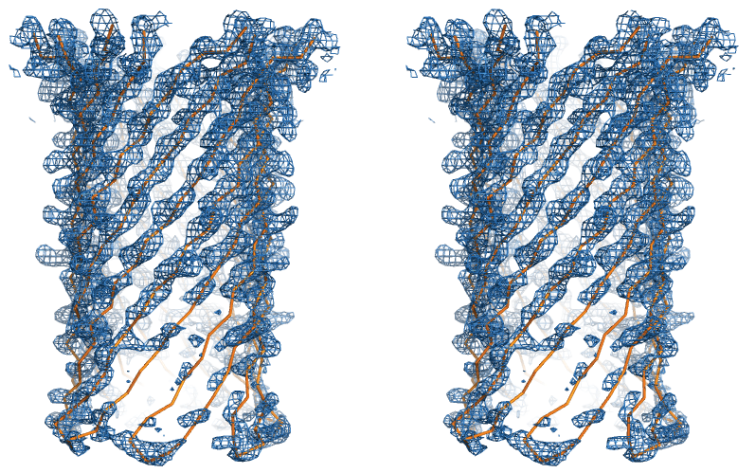

Fig. 3 (Sugawara et al.) 
A

amino latch (monomer)

D) amino latch (pore)
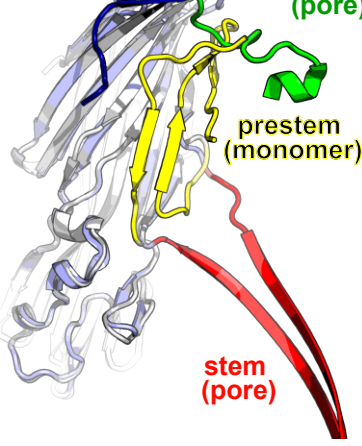

B

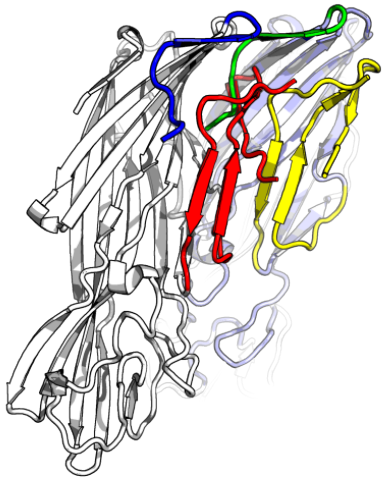

amino latch (monomer)
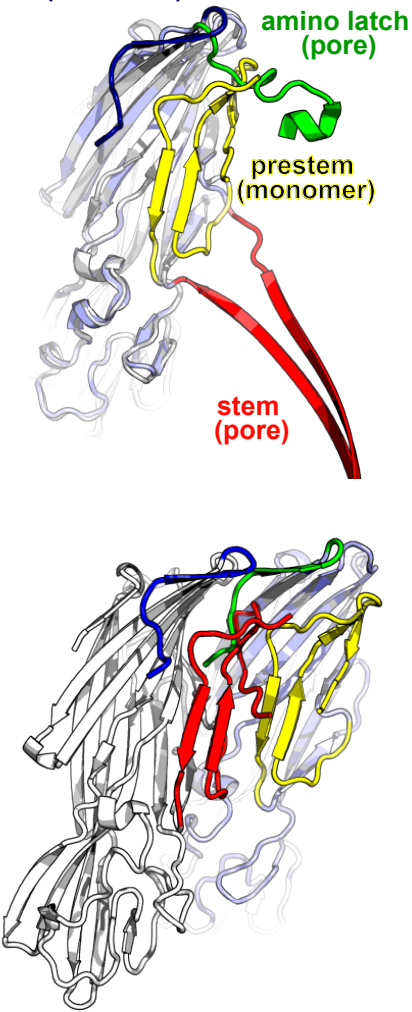

Fig. 4 (Sugawara et al.) 
A

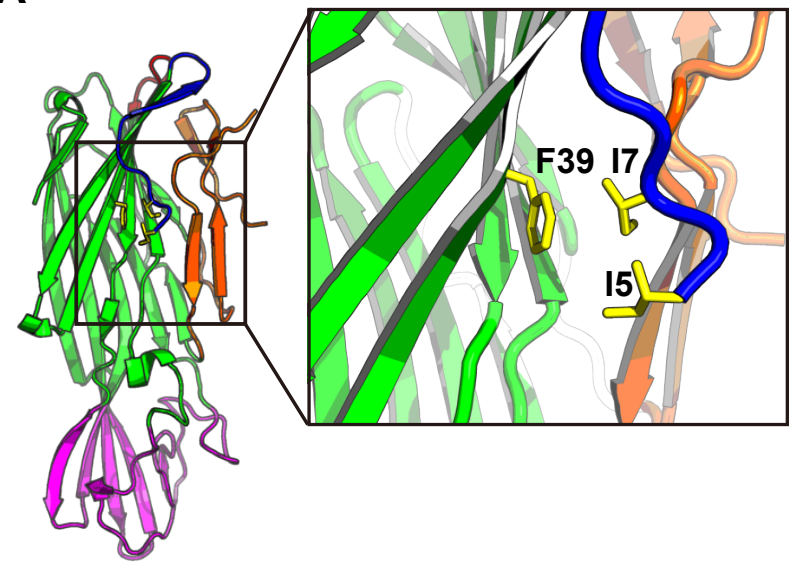

B

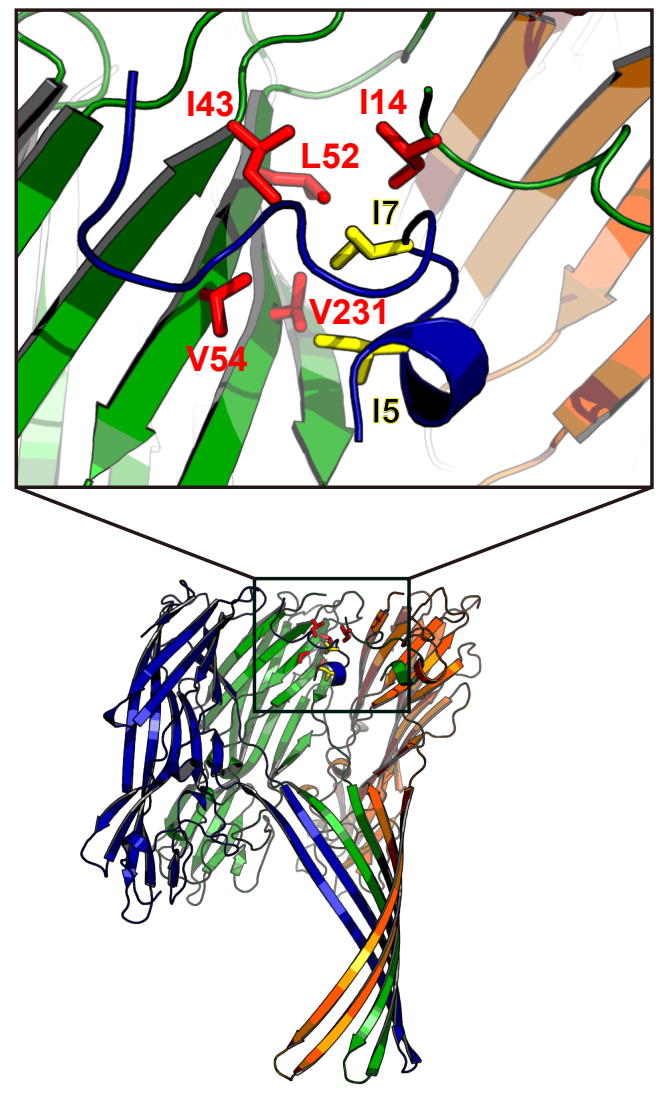

Fig. 5 (Sugawara et al.) 
Hla

LukF

LukFPV

LukD

Hlg2

LukSPV

LukE

ADSDINIKTGTTDIGSNTTVKTGDLVTYDKENGMHKKVFYSFIDDK . . EGKITPVSVKKVDDKVTLYKTTATADSDKFKISOILTF NFIKDK . AQH I TPVSEKKVDDKITLYKTTATSDSDKLKISO ILTF NFIKDK . AQH I TPVSEKKVDDKITLYKTTATSDNDKLNISQILTFNFIKDK ...... ENKIED I GQGAE I I KRTQDITSKRLAITQNIQF DFVKD ...... DNNIENIGDGAEVVKRTEDTSSDKWGVT QNI QF DFVKDK ....... NTNIENI GDGAEVIKRTEDVSSKKWGVTQNVQF DFVKDK

50 60 70

80

Hla LukF LukFPV LukD Hlg2 LukSPV LukE

90 100 110 120 130

Hla LukF LukFPV LukD Hlg2 LukSPV LukE LPDNEVAQ I SDYYPRNS I DTKEYMSTLTYGF NGNVTGDDTGKIGGI SQSNDSVNVVDYAPKNQNE EF QVONTLGYTF GEDIS . . ISNGLSGG SDSNDSVNVVDYAPKNQNEEFQVQQTVGYSYGGDIN . I ISNGLSGG SESNDAVNVVDYAPKNQNEEF $Q V Q Q T L G Y S Y G G D I N$. . I SNGLSGG TKDSN . VDL I NYLPKNK I DSADVSQKLGYNI GGNFQ . . SAPS IGE. TNDPN. VDLINYLPKNKI DSVNVSQTLGYNI GGNFN . . SGPSTGG TKDPN • VSL I NYLPKNKI ETTDVGQTLGYNI GGNFO . . SAPS IGG.

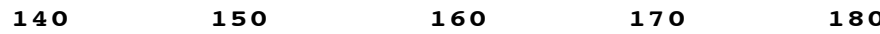

Hla LukF LukFPV LukD Hlg2 LukSPV LukE

I GANVS I GHTLKYVVPDFKTILES . PTDKKVGWKVIF NNMVNONWG LNGNTAF SETI NYKOESYRTTLSRNTNYKNVGWGVEAHKIMNNGWG GNGSKSFSETINYKOESYRTSLDKRTNFKKI GWDVEAHKIMNNGWG LNGSKSF SET I NYKOES Y RTTI DRKTNHKS I GWGVEAHK IMNNGWG . SGSFNYSKTI SYNOKNYVTEVES . QNSKGVKWGVKANSFVT . . . . NGSF NYSKTI SYNQQNY I SEVEH . QNSKSVQWG IKANSFIT . . . . NGSF NYSKTISYTOKSYVSEVDK . QNSKSVKWGVKANEFVT . . .

190

200

210

220

Hla

LukF

LukFPV

LukD

Hlg2

LukSPV

LukE

PYDRDSWNPVYGNQLFMKTRNGS . MKAAENFLDPNKASSLLSSGFS PYGRDSFHPTYGNELFLAGRQS A . YA GQNF IAQHQMPLISRSNFN PYGRDSYHSTY GNEMFL GSROSNL . NA GQNFLEYHKMPVLSRGNFN PYGRDSYDPTYGNELFL GGROSSS . NAGQNFLPTHOMPLLARGNFN PNGQVSAY DQY . . . LFAQDPTGP . . AARDYFVPDNQLP PLIQSGFN SLGKMSGHDPN ... LFVGYKPYSQ . NPRDYFVPDNELPPLVHSGFN PDGKKSAHDRY ...LFVQS PNGPTGSAREYFAPDNQLP PLVQSGFN
230
240
250
260

Hla

LukF

LukFPV

LukD

Hlg2

LukSPV

LukE

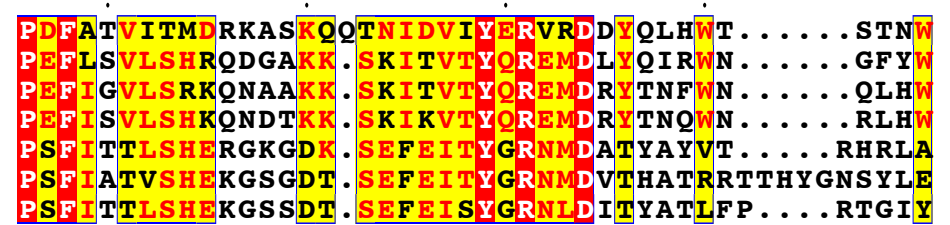

$270280 \quad 290$

Hla

LukF

LukF PV

LukD

Hlg2 KGTNTKDKWTDRSSERYKI DWEKEEMTN . . . . . . .
AGANYKNFKTRTF KSTYEI DWENHKVKLLDTKETENNK . I GNNYKDENRATHTS I YEVDWENHTVKL I DTOSKEKNPMS VGNNYKNONTVTFTSTYEVDWONHTVKLIGTDSKETNPGV

LukSPV DRKHDAFKNRNVTVKYEVNWKTHEVKIKSITPK.....

RIHNAF VNRNYTVKYEVNWKTHE I KVKGHN......

AERKHNAFVNRNF VVRYEVNWKTHEIKVKGHN . . . . . 